\title{
Zur Lehre von der physiologischen Thrombose der Uterusgefässe während der Schwangerschaft.
}

\author{
Von \\ Dr. F. Patenko. \\ (Aus der propädeutisch-gynäkologischen Klinik des Herro. Prof. Slavjansky \\ an der medico-chirurgischen Academie zu St. Petersburg.)
}

(Mit Abbildungen 'Tafel Ix.)

Im Jahre 1870 machte C. Friedländer die Beobachtung, dass gegen das Ende der Schwangerschaft sehr viele Gefässe des Uterus an der Placentarstelle durch eigenthümliche, aus grossen, schönen, den Decidualzellen ähnlichen Elementen bestehende Thromben verstopft werden. $\left.{ }^{1}\right)$ Nachdem er darauf noch, ,eine ganze Anzahl (?) neugesammelter Fälle ${ }^{\text {si }}$ untersucht, sagt er in seiner späteren Arbeit $\left.{ }^{2}\right)$; ,ich darf den beschriebonen Zustand mit der grössten Sicherheit als zu dem normalen Entwickelungsgange der Schwangerschaft gehörig auffassen."

Im Jahre 1877 stellte L e o pold ${ }^{3}$ ) Untersuchungen über diese Thatsachen an und bestätigte vollkommen die Behauptung Friedländer's in ihren Grundzügen; ausserdem führte er die Erklärung, welche Letzterer in Form einer Voraussetzung gab, weiter aus und bestätigte sie durch seine eigenen Präparate. Nach ihm beginnt der Process der Thrombose folgendermassen: „um eine oder mehrere, dicht beisammenliegende Venen sich feine, hellere 1870 .

1) Physiologisch - anatomische Untersuchungen über den Uterus, Leipzig

2) Ueber die Innenfläche des Uterus post partum, dieses Archiv, Bd. IX.

3) Studien über die Uterusschleimhaut während Menstruation, Schwangerschaft und Wochenbett. - Die spontane Venenthrombose in den letzten Monaten der Schwangerschaft. Dieses Archiv, Bd. XI, S. 492. 
Ringe als Gefässwand bilden, die eine homogene oder feinstreifige bis fibrilläre Grundsubstanz haben. Diese ist durchsetzt von einzelnen oder zahlreichen Riesenzellen, welche genau die Form und Grösse der aussen befindlichen haben und ebenso vollkommen übereinstimmen mit denen, welche das Gefässlumen in grösserer oder geringerer Anzahl erfüllen" (S. 496). Weiterhin behauptet Leopold: ,es bieten sich nirgends Anhaltspunkte oder Uebergangsformen dar, sie für Producte der Blutkörperchen oder der Gefässendothelien zu halten. Im Gegentheil sieht man, wie zuerst einzelne Riesenzellen rundlich oder gestreckt in den hellen Ringen liegen, dann der Intima anliegen und diese verschiebend mehr in das Gefässlumen hineingedrängt werden. Es kann sonach für diese Bilder (behauptet Leopold) nur die Erklärung geben, dass die Riesenzellen, welche man aus der Umgebung ganz unverändert fortgesetzt sieht, in die Uterusvenen einwandern."

Diese einfache Erklärung ruft jedoch vor Allem die natürliche Frage hervor: was ist die Ursache, welche Kräfte veranlassen diese grossen Obliterationszellen, zum Gefässe hinzuwandern, dessen Intima in das Gefässlumen vorzuschieben, die Zellelemente letzterer wahrscheinlich aus einander zu schieben und nach Ueberwindung eines bedeutenden Blutdruckes dennoch in das Gefäss einzuwandern? Allerdings lässt man ein Einwandern von Fremdkörpern in die Gefässe zu ( 0 . Weber $\left.{ }^{2}\right)$ ); allein erstens als eine seltene Erscheinung, und zweitens in pathologisch veränderte oder durch ulceröse Processe zerstörte, und nie in normale Gefässe, ,da im Allgemeinen die Druckverhältnisse des Kreislaufes einem. solchen Eintritte nicht günstig sind". Ueber das Eindringen von solchen Fremdkörpern, wie Eiter, in die Gefässe, finden wir bei Weber (l. c.): „Die alte Anschauung, welche noch jetzt in den Köpfen vieler Chirurgen spukt, wonach der Eiter ganz allgemein aus einem jeden Abscessherde direct durch die angefressenen klaffenden Venen aspirirt werden und Metastasen erregen könnte, ist längst widerlegt."

In Anbetracht Alles dieses haben wir uns zur Aufgabe gemacht, uns entweder von der Richtigkeit der Erklärung, welche Leopold auf Grund seiner eigenen Präparate giebt, zu überzeugen, oder eine mehr verständliche Erklärung der Thatsache zu

1) Handbuch der allgemeinen und speciellen Chirurgie von Pith a und Billroth, Bd. I, S. 82. 
finden, da diese Frage in der That, wie Leopold sagt, von grösstem Interesse ist.

Da die Thatsache der Thrombose der Uteringef ässe an der Placentarstelle selbst keinem Zweifel unterliegt, so erlaubten wir uns nur ein sehr geringes Material zu gebrauchen, in der Ueberzeugung, an diesem die Thatsache selbst aufzufinden. und vielleicht auch eine Erklärung dazu.

Diese äusserst schönen Zellenthromben trafen wir zuerst bei der Untersuchung des Uterus, welcher bei der Section der 35 Jahre alten Bäuerin P. J. erhalten wurde. Letztere wurde am 9. September 1878 mit einer ausgedehnten Verbrennung ron mehr als dreiviertel der Körperoberfläche, welche sie sich bei der Pulverexplosion auf der Patronenfabrik zugezogen, in die Klinik aufgonommen. Sie hatte drei zeitige Geburten durchgemacht, ein Mal abortirt. Anamnese und Exploration zeigten, dass sie sich im sechsten Monate der Gravidität befinde.

Ausserdem bedienten wir uns noch zweier puerperaler Uteri, welche im Museum der Klinik des Herrn Prof. Slavjausky sub Nr. 38 und 82 aufbewahrt sind.

Nr. 38. Dieses Präparat ist von einer 38jährigen Kreissenden P. J. erhalten, welche nach dem 39. Eclampsieanfalle starb ${ }^{1}$ ); die 39 Anfälle hatte sie während $121 / 2$ Stunden durchgemacht. Sie war das zwölfte Mal schwanger (zeitige Geburt). Accouchement forcé zehn Minuten post mortem. Ruptur des Collum uteri bis zum Orificium internum. Ruptur der Fornix bis zum Zellgewebe des Ligamentum latum.

Nr. 82. M. B., Bürgersfrau, 31 Jahre alt. Wurde am 1. Mai 1869 in das Entbindungszimmer der Klinik aufgenommen; starb am 2. Mai an einer Uterinblutung post partum, wie es im Sectionsprotocoll des hiesigen pathologisch-anatomischen Institutes vorn 3. Mai 1869 sub Nr. 193 heisst. Da wir die resp. Krankengeschichte nicht besitzen, kann ich Ausführlicheres nicht mittheilen.

Die durch Alcohol entwässerten Präparate wurden mit Pikrinsäure "bearbeitet, auf 24 Stunden in eine Lösung von Gummi arabicum eingebettet und darauf wieder in starken (95 ) Alcohol gebracht; zur Tinction der Schnitte diente Pikrocarmin und neu-

1) Bericht der Gebäranstalt des kaiserlichen Findelhauses, 1840-1871, S. 104. St. Petersburg 1872 (russisch). 
trales Carmin. Die so angefertigten Präparate zeigten bei einer Vergrösserung von $400 \mathrm{Mal}$ (Hartnack, Ocul. 3, System 8) schon in den äussersten Muskelschichten des Uterus eine deutliche Alteration der Gefässendothelien (Zeichnung 1 und 2): nicht etwa eine dünne, doppeltconturirte Platte mit kleinen localen Erhabenheiten, den Kernen des normalen Endothels entsprechend, sondern eine deutliche Zellenschicht mit in das Gefässlumen stark hervortretenden Kernen. Je mehr nach innen, d. h. in den innersten Muskelschichten, desto deutlicher tritt dieses Aufquellen des Endothels hervor; zuweilen sieht man hier and da eine Lostrennung desselben von den Gefässwandungen, im Gefässlumen selbst findet man aber inmitten des Blutpfropfes mit entfärbten Blutkörperchen dunkelgranulirte Zellen von bedeutender Grösse mit deutlich hervortretenden Kornen; letztere liegen bald einzeln und zerstreut im Blutpfropf, bald nehmen sie, sich anhäufend, einen beträchtlichen Theil des Gefässlumens ein (Zeichnung 3 und 4). Noch weiter nach innen finden wir eine noch bedeutendere Modification des Endothels: die Elemente sind noch mehr vergrössert (Zeichnungen 5 und 6), was besonders deutlich auf solchen Präparaten zu sehen ist, wo ausser quer durchschnittenen Zellen sich Endothelien en face darbieten, wie z. B. auf dem Präparate 6. Hier erinnern diese grossen, körnigen Elemente mit deutlich hervortretenden Kernen schon stark an Deciduazellen.

In den an die Decidua grenzenden Muskelschichten begegnen wir einem noch bedeutenderen Aufquellen des Endothels, und ausserdem finden sich in kleineren Gefässen Zellenconglomerate mit undeutlichen Grenzen (Zeichnung 7), da die Elemente dicht aneinander liegen; ihre Kerne jedoch, von der Grösse eines grossen Endothelkernes, sind immer deutlich zu sehen. In einem Falle fanden wir in der an die Decidua dicht angrenzenden Schicht ein kleines Gefäss vollständig durch dergleichen Conglomerate obliterirt (Zeichnung 9). Neben solchen Gefässen finden wir in der angrenzenden Schicht grosse Gefässe, deren Endothel in dem Maasse vergrössert ist, dass man deutlich sehen kann, was mit ihm geschieht: auf Zeichnung 8 ist ein Theil eines grossen, mit Blutpfropfen verstopften Gefässes zu sehen; an der inneren Wandung liegen grosse körnige Endothelzellen mit 2, 3 and $4 \mathrm{Ker-}$ nen; im Blutpfropf sehen wir Zellen von verschiedener Grösse, von einem weissen Blutkörperchen bis zu der Grösse einer grossen Endothelzelle. Einige Gefässe enthalten nur sehr wenig von diesen Zellen, andere in bedeutender Menge, und schliesslich finden 
wir Gefässe vollkommen obliterirt durch schöne, grosse, den Deciduazellen in der That ähnliche Elementen. Solche Gefässe besitzen eine deutlich ausgeprägte, wenn auch, im Vergleiche zu ihrer Grösse, etwas verdünnte Wandung; das Endothel der Intima bietet untrügliche Proliferationserscheinungen dar.

In der Grenzschicht der Decidua beobachtet man als Fortsetzung solcher obliterirten Gefässe Höhlungen von verschiedener Grösse, vollständig ausgefüllt von Zellentbromben. Dieses sind grosse, dünnwandige Capillargefässe, welche an den Stellen, wo der Thrombus herausgefallen, deutliche Proliferationserscheinungen des Endothels zeigen. Ausser diesen Zellenthromben sahen wir auch die von Friedländer erwähnten parietalen Thromben; diese erscheinen in der Form ron pilzähnlichen Excrescenzen, welche an der Innenfläche der Intima sitzen.

Neben derartig obliterirten Gefässen findet man auch, dem Augenscheine nach, vollkommen normale, und sind sie grösstentheils mit Blut überfiillt.

Somit finden wir eine Alteration einiger Uteringefässe, welche besonders scharf an der Placentarstelle hervortritt; diese Alteration beginnt mit dem Aufquellen, der Vergrösserung des Endothels, wird stärker mit der Annäherung an die Innenfäche des Uterus und endigt mit Zellenthromben an der Placentarstelle.

Indem das Gefässendothel bedeutend an Grösse zunimmt und in Folge dessen stärker in das Gefässlumen hervortritt, verursacht es in solchen Gefässen augenscheinlich eine beträchtliche Unebenheit auf der Innenfläche, und erscheint somit als eine Ursache für die Verlangsamung des Blutstromes und dio Gerinnung desselben. $\left.{ }^{1}\right)$ Dieses können wir in der That auch beobachten: je mehr wir uns der Innenfläche des Uterus nähern, je bedeutendere Veränderungen das Endothel zeigt, desto beständiger finden wir solche Gefässe verstopft; dabei befinden sich die älteren Pfröpfe in der Grenzschicht der Decidua und der anliegenden Muskelschicht des Uterus, wodurch die Annahme gerechtfortigt wird, dass gerade in diesen Schichten das Endothel zuerst die geschilderten Veränderungen durohmacht.

Somit sehen wir, dass ein Theil der Gefässe durch Blutpfröpfe verstopft wird. Bekanntlich unterliegen solohe Pfröpfe

1) Lehrbuch der pathologischen Gewebelehre von Rindfleisch, 1873, 3. Auflage. 
einer zweifachen Veränderung: entweder der Organisation oder der Erweichung. Bei der Organisation geht der Thrombus in Bindegewebe über. Weiter wissen wir nun, dass das in der Decidua befindliche Bindegewebe sich während der Schwangerschaft stark verändert: ihre Zellen nehmen bedeutend an Grösse zu, proliferiren energisch und häufen sich hier in grosser Menge an (Friedländer, l. c.). In einer so energisch wuchernden Nachbarschaft kann sich offenbar das junge Bindegewebe des sich organisirenden Thrombus dem Einflusse der Ursachen nicht entzichen, welche die charakteristischen Veränderungen in dem sie umgebenden älteren Bindegewebe hervorrufon. Die Bindegewebszellen im Thrombus, welche, wie bekannt, nach einigen Autoren aus den weissen Blutkörperchen, nach anderen auch aus dem Gefässendothel entstehen, nehmen an Zahl und Grösse zu, und zwar auf Kosten des proliferirenden Endothels, welches ebenfalls bindegewebigen Ursprunges ist. Auf diese Weise häuft sich in den Gefässpfröpfen eine beträchtliche Menge gerade desselben Materiales, aus welchem auch die grossen Deciduazellen hervorgehen, so dass es diese letzteren nicht im Geringsten nöthig haben, aus dem angrenzenden Bindegewebe zu dem Zwecke einzuwandern, um daselbst einen Thrombus zu bilden. Der sich organisirende Gefässpfropf hat also selbst Bindegewebselemente in hinreichender Menge; indem sich diese nun den in der Umgebung obwaltenden Bedingungen fügen, fangen sie an zu proliferiren, darauf sich $\mathrm{zu}$ vergrössern, und gehen schliesslich in die grossen Zellen über, welche die Gefässe verstopfen; dass sie nun den Deciduazellen gleichen, ist kein Wunder, da sie ihre Entstehung, wie denselben Elementen, so auch derselben Ursache verdanken.

Diese Ansicht über die Entstehung der aus Zellen bestehenden Gefässpfröpfe scheint uns verständlicher und einfacher zu sein, als die Leopold's. Unsere Ansicht können wir auch damit bekräftigen, dass ein ähnlicher Vorgang schon beschrieben ist. Das Verwachsen der Höhlung eines geplatzten, das befruchtete Ei liefernden Follikels, wie auch der nicht geborstenen, zu Grunde gehenden Follikelhöhlen während der Schwangerschaft, ist gerade durch Hypertrophie des pexifolliculären Gewebes bedingt, dessen Gewebe nach Slavjansky's ${ }^{1}$ ) Untersuchungen aus reticulärem Bindegewebe besteht, und welches ,Zellen von verschiedener Grösse enthält, von der Grösse eines weissen Blutkörpers an bis

1) Archives de Physiologie 1874, S. 219. 
zu der Grösse der Riesenzellen" (M. L. de Sinéty $\left.{ }^{1}\right)$ ). Im Laufe der Schwangerschaft nimmt die Hypertrophie dieser Schicht fortwährend zu: im sechsten Monate macht letztere zwei Drittel, gegen das Ende der Schwangerschaft aber drei Viertel der ganzen Masse des Corpus luteum aus (Sinéty, 1. c.).

Ebensowenig Unwahrscheinliches ist in der activen Theilnahme des Gefässendothels an der Bildung des Thrombus zu sehen. Wir können uns dabei sowohl auf unsere Präparate berufen, welche dieses mit grosser Evidenz beweisen, als auch auf den Umstand, dass seit dem Jahre 1877 von vielen Forschern ein actives Verhalten des Endothels bei vielen Vorgängen, welche in Folge von Ernährungsstörungen in irgend welchem. Theile des Organismus stattfinden, constatirt worden ist (K. Winogradoff $\left.{ }^{2}\right)$ ). Schliesslich geht bekanntlich die Fähigkeit des Endothels, auf Reize zu reagiren, so weit, dass es zur Quelle von Neubildungen - dor sogenannten Pseudoepitheliome oder Endotheliome - werden kann.

Es wäre hier am Platze, zu erwähnen, dass sich Leopold, wie es uns scheint, zu seiner oben erwähnten Erklärung nicht normaler, sondern pathologisch veränderter Gefässe bedient habe. Wir fanden nämlich auf den Präparaten vom Uterus Nr. 38 solche Gefässe, welche „, von Ringen eines hellen Gewebes aus homogener Substanz"6 umgeben sind. Ein Theil eines solchen Gefässes ist auf Zeichnung 11 abgebildet. Nach der Beschreibung I eopold 's müssen das eben die Gefässe sein, an denen er das Einwandern dex Deciduazellen in das Gefässlumen durch die Gefässwandung hindurch bewiesen haben will. Allein ein Blick auf die beigegebene Zeichnung (11) genügt, um zu sagen, dass wir es hier mit einer Perivasculitis (Slavjansk $\mathrm{y}^{3}$ )) zu thun haben: dieser Process hat nicht nur die Gefässwandung ergriffen, sondern auch das perivasculäre Bindegewebe, so dass der helle Gefässring unmittelbar an der Muskelschicht anliegt. Die in diesem hellen Ringe liegenden grossen Zellen sind offenbar nichts anderes, als hypertrophirte Bindegewobszellen (Ranvier) der Gefässwandungen. Es ist nun sehr wahrscheinlich, dass auch Leopold etwas Aehnliches angetroffen, da dieser pathologische Forgang, nach Slavjansky's (1, o.) Untersuchung, im Uterus nicht selten vorkommt.

1) De l'ovaire pendant la grossesse; Comptes rendus 1877.

2) Ueber das Pseudo-epitheliom, Zeitschrift für normale und pathologische Histologie, red. von Prof. Rudnew, Bd. IX, 1875 (russisch).

3) Endometritis villosa, Archives de Physiologie 1874, 2me Série. 
Wir haben ihn schon einmal im Uterus bei Graviditas ovarialis ${ }^{1}$ ) beschrieben und begegnen ihm wieder in einem der drei Uteri, die wir untersuchten.

Somit erlauben wir uns, auf Grund unserer Untersuchung 1) die Angabe Friedländer's über das Vorkommen von Zellpfropfen an der Placentarstelle zu bestätigen; und 2) behaupten wir, dass diese Zellpfröpfe ein Product der Proliferation und $\mathrm{Hy}-$ pertrophie der Bindegewebselemente sind. Die Organisation des Thrombus geht hier ebenso vor sich, wie an anderen Stellen des Organismus; der grössere Reichthum an Zellelementen rührt von einer stärkeren Proliferation des Endothels der Intima her. Erst später gehen die Bindegewebszellen dieser Gefässpfröpfe auf dem Wege der Hypertrophie in grosse Zellen über, welche, stellenweise sich anhäufend, die Gefässe verstopfen.

Eine derartige Erklärung des Vorganges scheint auch a priori wahrscheinlicher zu sein.

St. Petersburg, am 20. Februar 1879.

\section{Erklärung der Zeichnungen.}

1. Querschnitte von Gefässen aus der äusseren Muskelschicht der

2.) Gebärmutterwandung.

3. Id. aus den mittleren Schichten.

5. Id. aus den inneren Schichten.

7. Aus der inneren, an die Decidua grenzenden Muskelschicht.

8. Theil eines grossen Gefüssses aus derselben Region.

9. Aus der Grenzschicht der Decidua.

10. Zellthrombus aus der angrenzenden Muskelschicht.

11. Theil eines Gefässes aus derselben Region mit pathologisch veränderten Wandungen (Perivasculitis).

1) Dieses Archiv, Bd. 14, Heft 1. 

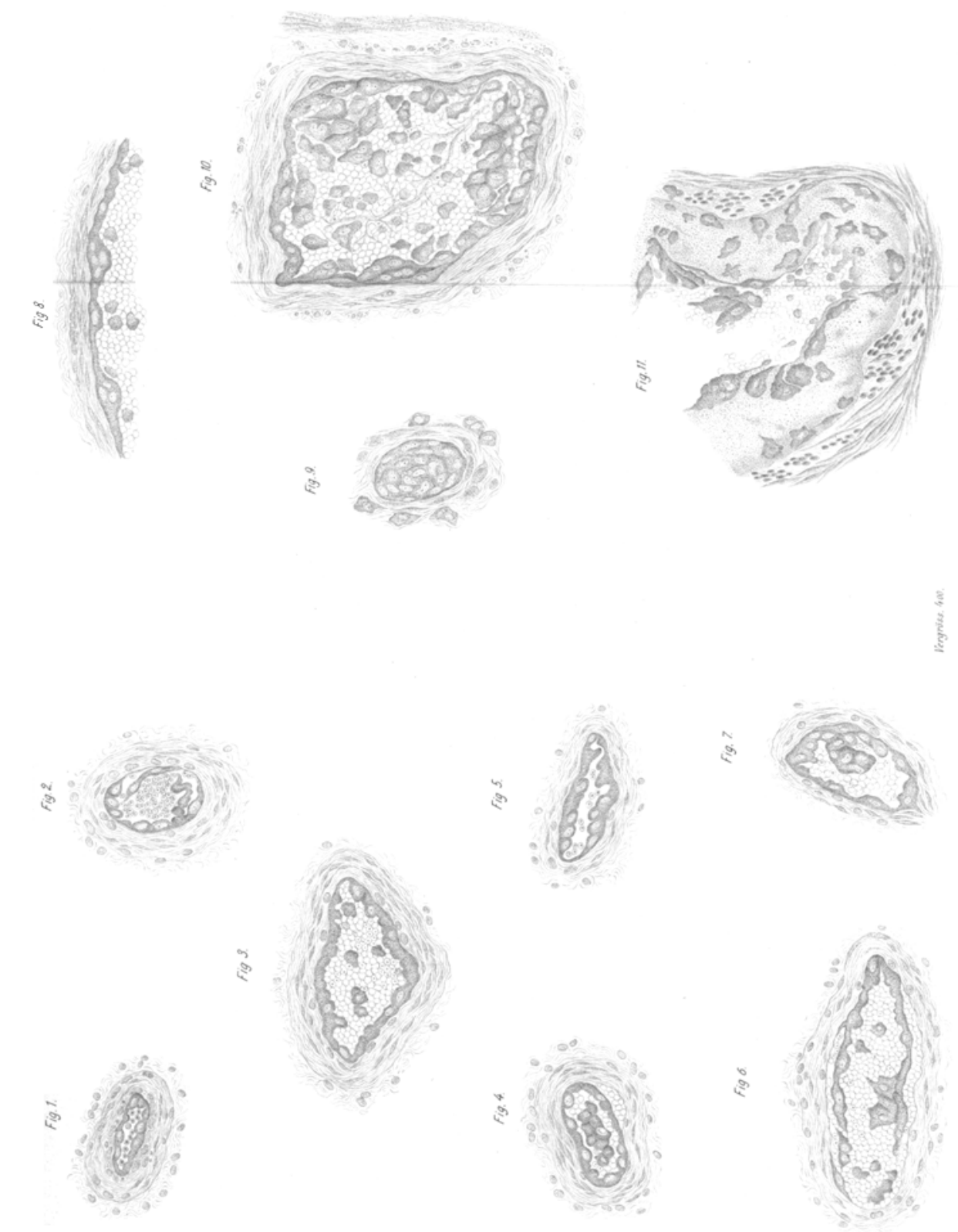